The manuscript for Applied Microbiology and Biotechnology (Original papers)

\title{
Cloning and characterization of a gene coding for a hydrophobin Fv-hyd1 specifically expressed during fruiting body development in the basidiomycete Flammulina velutipes
}

Masato Yamada, Sou Sakuraba, Kou Shibata, Satoshi Inatomi,* Mitsuo Okazaki, and Makoto Shimosaka

Department of Applied Biology, Faculty of Textile Science and Technology, Shinshu University, 3-15-1 Tokida Ueda, Nagano 386-8567 Japan

*Mushroom Research Laboratory, Hokuto Corporation, 800-8 Shimokomazawa, Nagano 381-0008, Japan

Communicating author:

Makoto Shimosaka

e-mail: mashimo@shinshu-u.ac.jp

Tel \& Fax: +81-268-21-5341 
Abstract A cDNA of hydrophobin (fv-hyd1), which is specifically expressed during fruiting body development, was isolated from the basidiomycete Flammulina velutipes by a differential display screening. The analysis of the genomic structure of fv-hyd1 revealed an open reading frame (ORF) composed of 363 bp-nucleotides and interrupted by three introns. The deduced amino acid sequence of FV-HYD1 showed a similarity to those of other fungal class I hydrophobins and contained eight cysteine residues highly conserved among hydrophobin proteins. The pattern of the hydropathy plot of FV-HYD1 was similar to those of class I hydrophobins. The Southern blot analysis of genomic DNA showed that fv-hyd1 existed as a single copy. Northern blot analysis indicated that the $f v$-hyd1 transcript was not present in vegetative mycelia but markedly increased in level at the primordial stage. Moreover, the fv-hyd1 transcript was abundant even at the mature-fruiting-body stage. This result indicates that fv-hyd1 would encode a hydrophobin closely associated with fruiting body development.

\section{Introduction}

Mushrooms (fruiting bodies of basidiomycetes) have long been used as foods worldwide. Some mushrooms contain unique metabolites with antitumor or immunomodulating activity and have been used as medicine. Moreover, basidiomycetes have an ability to degrade lignin and cellulose in wood and have been examined for utilization in biomass conversion (Kues and Liu 2000). At present, only limited types of basidiomycetes are cultivated for the commercial production of mushrooms, which are used as food or as a source for the extraction of useful metabolites. However, most basidiomycetes including those with a high commercial value cannot be cultivated or induced to fruit in artificial culture. This difficulty is mainly ascribed to our poor understanding of the process of fruiting body development in terms of molecular biology or molecular genetics (De Groot et al., 1996).

In a commercial-scale production of mushrooms, the development of fruiting bodies is induced by controlling various environmental factors such as light, temperature, humidity, physical stimulus, and chemical stimulus. These factors likely promote the expression of 
crucial gene(s) such as transcription factors, which in turn triggers a set of genes indispensable for the initiation of fruiting. The study of genes involved in this initiation of fruiting is of interest to clarify a fundamental process of fruiting at the molecular level and will also help us to develop an efficient and reproducible method of cultivating and inducing the development of fruiting bodies of various valuable species of basidiomycetes.

We have been studying fruiting body development using an edible basidiomycete, Flammulina velutipes (called enokitake in Japan). F. velutipes has long been cultivated industrially and fruiting can be induced reproductively by a combination of physical stimuli (e.g., scraping off the mycelial mat on the medium surface, water pouring, and lighting). A well-synchronized emergence of primordia (young fruiting bodies) indicated that F. velutipes is a good material for the analysis of genes functioning at an initial stage of fruiting. We already isolated 610 cDNA fragments that were not expressed in vegetative mycelia but significantly expressed at the stage of primordium formation by a differential display screening (in preparation for publication). Some of these cDNAs are expected to code for a protein that plays a crucial role in an initial stage of fruiting. Sequence analysis revealed one cDNA fragment whose sequence showed a significant similarity to those of genes encoding fungal hydrophobins.

Hydrophobins are fungal-specific small hydrophobic proteins that are characterized by the presence of eight conserved cysteine residues. They are secretory proteins and self-assemble into amphipathic layers at hydrophilic-hydrophobic interfaces. Fungal strains usually contain several genes coding for hydrophobins, which correspond to their divergent functions such as the formation of aerial structures, mediation of hyphal attachment on hydrophobic surfaces, and formation of hydrophobic rodlet layers on a fungal spore (Wosten et al., 1999). Hydrophobins are composed of four loops, which are formed by disulfide bonds among eight cysteine residues that are highly conserved. Hydrophobins are grouped into two classes (classes I and II) due to their solubility in various solvents (Wessels 2000). All reported hydrophobins from basidiomycetes belong to class I of hydrophobins. In basidiomycetes, genes coding for hydrophobins are developmentally regulated. Some of these genes are highly 
expressed in fruiting bodies, and thus encoded hydrophobins would be closely associated with the morphology of fruiting bodies (Wessels 1997). In this paper, we report an analysis of a novel hydrophobin gene isolated from F. velutipes and describe its transcriptional regulation during the process of fruiting body development.

\section{Materials and methods}

Strain and cultivation conditions

A commercially cultivated F. velutipes dikaryotic strain MH092086 was used for the isolation of cDNAs that are specifically expressed during fruiting. A sawdust medium was composed of Cryptomeria sp. sawdust (17.5\%, w/w), corncob meal (11\%), rice bran (10\%), and water (61.5\%). The medium was contained in an 850 ml-culture bottle (polypropylene) and sterilized at $120{ }^{\circ} \mathrm{C}$ for $40 \mathrm{~min}$. The capped bottles inoculated with mycelial plugs were incubated at $15{ }^{\circ} \mathrm{C}$ for 30 days in the dark until vegetative mycelia grew all over the medium. Then fruiting was induced by applying a combination of three stimuli, namely, physical stimulation (scraping off the mycelial mat on the medium surface), addition of $20-30 \mathrm{ml}$ of water, and exposure to white fluorescence light (50 lux) at $15^{\circ} \mathrm{C}$ (90\% humidity). Primordia (young fruiting bodies, 2-5 mm in length) appeared after 10 days of induction. For the maturation of fruiting bodies, the bottles were incubated at $8{ }^{\circ} \mathrm{C}$ under continuous light (50 lux) for another 20 days. Vegetative mycelia (immediately before induction), primordia, and fruiting bodies were collected, immediately frozen in liquid nitrogen, and stored at $-80{ }^{\circ} \mathrm{C}$ until use.

Preparation of total RNAs and northern blot analysis

Frozen cells were homogenized to a fine powder in liquid nitrogen with a mortar and pestle.

Then total RNAs were prepared using TRI reagent (Molecular Research Center, Inc.) according to the manufacturer's protocol. To remove genomic DNAs contaminating RNA preparations, samples were treated with RNase-free DNase I (TaKaRa). Total RNAs were denatured and fractionated by agarose-formaldehyde gel electrophoresis, and transferred onto 
a GenScreen Plus membrane (NEN Life Science Products). Probe DNA labeling, hybridization, and signal detection were carried out using an ECL Direct Nucleic Acid Labeling and Detection system (Amersham). Chemifluorescent signals were visualized and quantified using an imaging analyzer STORM860 (Amersham). The size of transcripts was estimated by comparing with RNA molecular weight markers (Roche).

\section{Preparation of genomic DNAs and Southern blot analysis}

Genomic DNAs of F. velutipes dikaryotic or monokaryotic strains were prepared from mycelia grown on the potato-dextrose agar medium (Difco) according to the method previously described (Garber and Yoder 1983). The prepared DNAs were digested with Eco RV or Sal I and fractionated by agarose gel electrophoresis. Separated products were transferred onto a GenScreen Plus membrane (NEN Life Science products). Hybridization analysis was carried out in a similar manner to northern analysis.

Fluorescence differential display

Total RNAs prepared from vegetative mycelia (before fruiting induction) and primordia (10 days after induction) were used to screen a group of genes expressed only in the latter. First-strand cDNA synthesis, PCR amplification, and detection of amplified products after polyacrylamide gel electrophoresis were carried out using a fluorescence differential display (FDD) kit (Takara) and FMBIO II (Takara) according to the manufacturer’s protocol. We used a combination of nine fluorescein isothiocyanate (FITC)-labeled downstream primers and 12 upstream primers (total, 108 PCR sets) and screened for fluorescent cDNA bands appearing on polyacrylamide gels only for the primordial sample by comparing amplified cDNAs from both mycelial and primordial samples on the same gel. These primordium-specific cDNA fragments (300-1500 bps in size) were cut from the gel, extracted, and re-amplified using the non-FITC-labeled primer set that was used for the first PCR. The resulting amplified DNA fragments were ligated into the pT7Blue vector (Novagen) and propagated in Escherichia coli JM109. 


\section{General DNA manipulation}

Conventional DNA recombination techniques were used for DNA manipulation. A genomic DNA library was constructed using partially Sau 3AI digested chromosomal DNA and a Bam HI-digested Lambda DASH II vector (STRATAGENE). The library consisted of $3.9 \times 10^{4}$ phage clones with insert sizes of 9-23 kbp. The nucleotide sequence was determined by the dideoxy termination method using a DNA sequencer (Applied Biosystems, Genetic Analyzer 310). Sequence data were analyzed with Genetyx software (Software Development). The nucleotide sequence of the hydrophobin gene ( $f v$-hyd1) determined in this work has been deposited in the DDBJ database under accession number AB12668.

\section{Results}

Isolation of a hydrophobin-like gene from $F$. velutipes

We isolated 610 partial fragments of cDNAs that were expressed at the primordial stage during the fruiting process but not in the vegetative mycelium by differential display screening. The determination of the nucleotide sequences of cDNAs chosen at random revealed one cDNA clone (named 45c, 340 bp in length) that showed a significant similarity to those of fungal genes coding for hydrophobins. The genomic library was screened to obtain a full-length gene using the 45c cDNA fragment as a probe. As a result, eight clones were isolated and purified from approximately 20,000 plaques of the library after two successive screening by plaque hybridization. Phage DNAs prepared from these eight clones were digested with Eco RI and subject to Southern blot analysis using the 45c cDNA fragment as a probe. A single positive signal of $7 \mathrm{kbp}$ in size was detected from all of the eight clones (data not shown). Moreover, DNA fragments of identical size could be PCR-amplified from the phage DNAs of all eight clones when a set of PCR primers based on the sequence of the 45c cDNA fragment was used. These results clearly indicate that a gene corresponding to the $45 \mathrm{c}$ cDNA fragment was successfully isolated from the genomic library. A hybridization signal-positive 7-kbp-Eco RI fragment was purified from one of the clones and ligated into 
the pUC119 vector. The restriction map of this 7-kbp-DNA fragment is shown in Fig. 1.

Sequence analysis of hydrophobin gene (fv-hyd1)

A 900-bp nucleotide sequence of a portion of the inserted DNA was determined by a gene walking technique starting from the predetermined sequence of the 45c cDNA fragment (Fig. 1). A cDNA fragment encompassing the entire putative ORF was amplified by reverse transcriptase (RT)-PCR and its sequence was also determined. Comparison between genomic and cDNA sequences revealed a single ORF composed of $363 \mathrm{bp}$, which was interrupted by three small introns of 56, 61 and 55 bp (Fig. 2). These three introns contain the 5' and 3' border sequences that are typical of introns in the genes of filamentous fungi (Breathnach and Chambon 1981). In the 5' region upstream of ORF, one TATA box (TATAAG) at position -100 to -94 and three CT-rich motifs (at positions -170 to -143 , -64 to -53 and -47 to -30 ) were found. Downstream of ORF, a polyadenylation signal (ATATACAAA) was located at position 618 to 626 . The sequence of the 3' end of the 45c cDNA revealed a poly(A) tail at a position of 692. There are two in-frame ATGs (at positions of 1-3 and 34-36) that will fit for a start codon in the predicted ORF. A distance between the TATA box and the start codon is highly conserved in the hydrophobin genes of basidiomycetes. This distance is usually approximately $100 \mathrm{nt}$ as exemplified by $103 \mathrm{nt}$ in Schizophyllum commune Sc3 (Schuren and Wessels 1990) and 105 nt in Agaricus bisporus abh1 (De Groot et al., 1996). Considering this information, the first ATG (position 1-3) could be assigned to a start codon, which was located 100 nt downstream of the deduced TATA box. Moreover, a sequence in the vicinity of this ATG (TCATCATGAG) agreed well with the consensus sequence reported for fungal genes (Ballance 1986).

The predicted ORF would encode a protein of 121 amino acids with a calculated molecular mass of 11,969 Da. The analysis of the N-terminal amino acid sequence using SignalP software estimated a signal sequence composed of 21 amino acids with a high fidelity. The removal of this putative signal sequence yields a polypeptide of 100 residues with a molecular mass of 9,773 Da. The comparison of the deduced amino acid sequence of ORF with 
sequences in the database revealed significant degrees of similarity with those of hydrophobins from various basidiomycetes. The highest similarity was found with POH3 from Pleurotus ostreatus (65\% identity). Multiple alignments of these sequences of fungal hydrophobins clearly indicate that eight cysteine residues characteristic of hydrophobins were completely conserved in the deduced amino acid sequence (Fig. 3). Comparison of a hydropathy plot of the sequence with those of class I hydrophobins from other basidiomycetes demonstrated a high similarity in their patterns (Fig. 4). Thus, we referred to the isolated gene as the F. velutipes hydrophobin gene (fv-hyd1).

\section{Southern blot analysis of fv-hyd1}

Genomic DNAs were prepared from F. velutipes dikaryotic strain MH092086 (used in this work) and its parental monokaryotic strains, FB89 and MF38. The DNAs were completely digested with Eco RV or Sal I, whose recognition sites are not found in fv-hyd1, and subjected to Southern blot analysis using a part of the fv-hyd1 gene as a probe. A single hybridization signal of identical size (4.0 kb for Eco RV and $3.5 \mathrm{~kb}$ for Sal I) was detected from all of the three strains (Fig. 5). This result indicates that fv-hyd1 is a single-copy gene and is likely located in the same locus in the genomes of two monokaryotic strains that were used in the construction of the dikaryotic strain MH092086.

Expression of fv-hyd1 during fruiting body development To examine the expression of $f v$-hyd1 during one life cycle of $F$. velutipes, northern blot analysis was carried out using cells of the dikaryotic strain MH092086 obtained at different stages of fruiting body development. Total RNAs were prepared from cells of six different stages, that is, vegetative mycelial stage (immediately before the induction of fruiting), at 4 hr , on days 2, 6, 10 (primordial stage), and 16 (mature-fruiting-body stage) after induction. The total RNAs were fractionated by agarose gel electrophoresis, and separated products were transferred to a nylon membrane and hybridized with the fv-hyd1 probe. To confirm that equal amounts of RNAs were loaded in all lanes, the blot membrane was also hybridized with the $F$. 
velutipes $18 \mathrm{~S}$-rDNA probe. Any signal of the fv-hyd1 transcript was not detected in the vegetative mycelial stage, whereas a significantly high level of transcript (700 nt in length) appeared on day 10 after induction (primordial stage) (Fig. 6). This high level of transcript was maintained on day 16 when mature fruiting bodies developed. In this study, fruiting was induced by a combination of three different stimuli, the scraping-off of the mycelial mat from the medium surface, addition of water, and light exposure. To check which stimulus was the most effective in inducing fv-hyd1 transcription, we prepared three cultures and subjected them to only one of these three stimuli and examined the level of fv-hyd1 transcript by northern blot analysis on day 10 after the treatment. A significantly high level of transcript was observed in cells exposed to light, but the transcript could not be detected in cells subjected to mycelial mat scraping or water addition (Fig. 6).

\section{Discussion}

We isolated and determined the genomic structure of the fv-hyd1 gene that codes for a hydrophobin protein from the dikaryotic strain of $F$. velutipes. The fv-hyd 1 cDNA was initially obtained from a cDNA library that was constructed by differential display screening, targeting genes specifically expressed at the primordial stage during the fruiting process. Northern blot analysis confirmed that the $f v$-hyd1 gene was highly expressed at the primordial stage and mature-fruiting-body stage but not in vegetative mycelial stage (Fig. 6). Therefore, we assume that FV-HYD1 would be a fruiting body specific hydrophobin closely associated with fruiting body development.

In general, most basidiomycete species have multiple hydrophobin genes. For example, four hydrophobin genes (Sc1, Sc3, Sc4, and Sc6) have been isolated from S. commune (Van Wetter and coworkers 2000a, b). It was also reported that the expression of multiple hydrophobin genes is developmentally regulated in S. commune (Dons et al., 1984), A. bisporus (De Groot et al., 1999), and P. ostreatus (Asgeirsdottir et al., 1998, Penas et al., 1998). In the case of $S$. commune, the $S c 3$ gene is expressed both in monokaryons and dikaryons, whereas the Sc4 gene is expressed only in dikaryons (Van Wetter et al., 2000a). 
One hydrophobin gene (named fvh1) from F. velutipes was previously reported, which was specifically expressed after the induction of fruiting (Ando et al., 2001). The deduced amino acid sequence of FV-HYD1 shows a low similarity to that of FVH1 (only $44 \%$ identity), although eight-cysteine residues characteristic of hydrophobins are completely conserved in both sequences. The fvh1 gene was highly expressed in the vegetative mycelia one day after induction and during the initial stage of fruiting but not expressed in mature fruiting bodies. On the contrary to this, the fv-hyd1 gene was not expressed during the initial stage of fruiting (on days 2 and 6 after induction) but expressed in pre-mature (primordium) and mature fruiting bodies. Hence, the $f v$-hyd1 gene is a novel hydrophobin gene closely associated with the fruiting process. Southern blot analysis revealed that the fv-hyd1 gene is a single-copy gene in the genome of F. velutipes (Fig. 5). We could detect faint cross-hybridized signals that might correspond to other hydrophobin genes including fvh1. It is not surprising that the intensity of these signals were fairly low since the level of global identity among hydrophobin genes are very low. The developmental regulation of multiple hydrophobin genes in basidiomycetes is likely related to a variation of the basic structure of hydrophobins and therefore to their different functions in various stages of cell differentiation.

Most hydrophobin genes are abundantly transcribed in basidiomycetes. The analysis of a genomic structure of the $f v$-hyd1 gene revealed three CT-rich motifs as well as one TATA box in the region upstream of ORF (Fig. 2). These CT-rich motifs are located both upstream and downstream of the TATA box. Similarly, the F. velutipes fvh1 gene possesses CT-rich motifs on both sides of the TATA box (Ando et al., 2001). CT-rich motifs are often found immediately upstream of a transcription start point of genes that are abundantly transcribed in Neurospora crassa and Aspergillus nidulans (Gurr et al., 1988). Two hydrophobin genes of S. commune (Sc3 and Sc4) also have CT-rich motifs immediately before their transcription start points, and the transcript of Sc4 abundantly accumulated (Mulder and Wessels 1986, Schuren and Wessels 1990). We found that the transcription of fv-hyd1was highly promoted at the primordial stage and accumulated in a large amount (Fig. 6). Thus, CT-rich motifs found in the promoter region of hydrophobin genes may be recognized by some transcription 
activating factors and result in the high production of hydrophobins in a specified part or tissue of basidiomycetes.

Among fungal hydrophobin proteins, signal sequences usually vary highly in their sequences and lengths. The mature proteins of class I hydrophobins have eight cysteine residues that are highly conserved. The consensus rule for the number of amino acid residues between these eight cysteine residues was proposed as follows, -C- $\mathrm{X}_{6-7}-\mathrm{C}-\mathrm{C}-\mathrm{X}_{29-36} \mathrm{C}-\mathrm{X}_{11-13}-\mathrm{C}-\mathrm{X}_{5}-\mathrm{C}-\mathrm{C}-\mathrm{X}_{12-13}-\mathrm{C}-$ (Kershaw and Talbot 1998). According to this rule, the number of residues between the first cysteine and the second cysteine are 6 or 7 in class I hydrophobins reported to date. One exception for this consensus rule is A. bisporus ABH3, which has eight residues in this region (Lugones et al., 1998). Likewise, FV-HYYD1 that has nine residues does not conform to this consensus rule (Fig. 2). Hydrophobin is composed of four loops that are formed by disulfide bonds among the eight conserved cysteine residues. These loops constitute hydrophilic and hydrophobic regions in the molecule and play a role in self-assembly or attachment to hydrophobic surfaces (Wosten and De Vocht 2000). FV-HYD1 contains two extra amino acid residues in the first loop from the $\mathrm{N}$ terminus, but no significant differences were observed in the pattern of the hydropathy plot (Fig. 4). These two additional residues are likely to have no significant effects on the characteristic and function of a hydrophobin protein.

The development of fruiting bodies in basidiomycetes is induced by various environmental factors such as temperature, light and $\mathrm{CO}_{2}$ concentration. During this development, some signals or mediators would play a crucial role in triggering the expression of a set of genes that complete the entire fruiting process. However, the elucidation of these genes and their regulation has not yet been fully understood. Results of our present study clearly indicated that the fv-hyd1 gene encoding a novel hydrophobin is specifically expressed at the primordial stage and mature-fruiting-body stage (Fig. 6). To clarify the regulation of fv-hyd1 expression, we focused on the effect of the three stimuli (mycelial mat scraping, water addition, and light exposure) that were provided simultaneously for the induction of fruiting. When one of the three stimuli was applied, the transcript of fv-hyd1 was detected only in the light-exposed 
sample (Fig. 6). Few minute primordia were also observed on the medium surface in this sample, while no primordia were observed in the other two samples (data not shown). This result may imply that light exposure is a minimum requirement for the induction of fruiting in F. velutipes. Considering that fv-hyd1 was not expressed at the early stage of fruiting (at $4 \mathrm{~h}$, on day 2 and day 6 after induction), fv-hyd1 is not likely directly regulated by light exposure but by some preceding factors controlling the entire process of fruiting. As a result, the expression of fv-hyd1was initiated at the primordial stage (on day 10 after induction). Signaling pathways regulating expression of hydrophobins in basidiomycetes are still unclarified. The understanding of these pathways particularly including the effect of light exposure will provide valuable insights into the molecular mechanism underlying fruiting body development.

The expression of an other hydrophobin gene (fvh1) of F. velutipes was reported to be induced by mycelial mat scraping alone and the fvh1 transcript accumulated one day after the treatment (Ando et al., 2001). Therefore, it was speculated that FVH1 plays a role in the recovery from damage of mycelia following mycelial mat scraping. This expression pattern of fvh1is distinctly dissimilar from that of fv-hyd1, indicating that physiological roles of these two hydrophobins are totally different. A fruiting-body-specific hydrophobin SC4 of S. commune has been studied in detail and was shown to be localized abundantly on the surface of passages called air channels in fruit body tissues. This passage promotes an exchange of air between the inside and outside of the tissue, and the hydrophobic nature of hydrophobins localized on the surface is important in keeping away water from the channel (Lugones et al., 1999). Hydrophobins have various different functions, which are closely related to their primary structures. To examine the precise role of hydrophobin FV-HYD1 in fruiting body development, it is necessary to determine its cellular localization and its function in relation to cellular morphology. For this purpose, in situ hybridization analysis of fv-hyd1 transcripts and the examination of the effect of $f v$-hyd1 gene disruption on fruiting process are now undertaken. 


\section{Acknowledgements}

This work was supported by Grant-in-Aid for 21st Century COE Program by the Ministry of Education, Culture, Sports, Science, and Technology. We indebted to Division of Gene Research, Research Center for Human and Environmental Sciences, Shinshu University for providing facilities.

\section{References}

Ando A, Harada A, Miura K, Tamai Y (2001) A gene encoding a hydrophobin, fvh1, is specifically expressed after the induction of fruiting in the edible mushroom Flammulina velutipes. Curr Genet 39: 190-197

Asgeirsdottir SA, De Vries OMH, Wessels JGH (1998) Identification of three differentially expressed hydrophobins in Pleurotus ostreatus (oyster mushroom). Microbiology 144: 2961-2969

Ballance DJ (1986) Sequence important for gene expression in filamentous fungi. Yeast 2: 229-236

Breathnach R, Chambon P (1981) Organization and expression of eucaryotic split genes coding for proteins. Annu Rev Biochem 50: 349-383

De Groot PWJ, Schaap PJ, Sonnenberg ASM, Visser J, Van Griensven LJLD (1996) The Agaricus bisporus hypA gene encodes a hydrophobin and specifically accumulates in peel tissue of mushroom caps during fruit body development. J Mol Biol 257: 1008-1018

De Groot PWJ, Roeven RTP, Van Griensven LJLD, Visser J, Schaap PJ (1999) Different temporal and spatial expression of two hydrophobin-encoding gene of the edible mushroom Agaricus bisporus. Microbiology 145: 1105-1113

Dons JJM, Springer J, De Vries SC, Wessels JGH (1984) Molecular cloning of a gene abundantly expressed during fruiting body initiation in Schizophyllum commune. J Bacteriol 157: 802-808 
Garber RC, Yoder OC (1983) Isolation of DNA from filamentous fungi and separation into nuclear, mitochondrial, ribosomal, and plasmid components. Anal Biochem 135: 416-422

Gurr SJ, Unkles SE, Kinghorn JR (1987) The structure and organization of nuclear genes in filamentous fungi. In: Kinghorn JR (ed) Gene structure in eukaryotic microbes. IRL Press, Oxford, 93-139

Kershaw MJ, Talbot NJ (1998) Hydrophobins and repellents: proteins with fundamental roles in fungal morphogenesis. Fungal Genet Biol 23: 18-33

Kues U, Liu Y (2000) Fruiting body production in basidiomycete. Appl Microbiol Biotechnol 54: $141-152$

Lugones LG, Wosten HAB, Wessels JGH (1998) A hydrophobin (ABH3) specifically secreted by vegetatively growing hyphae of Agaricus bisporus (common white button mushroom). Microbiology 144: 2345-2353

Lugones LG, Wosten HAB, Birkenkamp KU, Sjollema KA, Zagers J, Wessels JGH (1999) Hydrophobin line air channels in fruiting bodies of Schizophyllum commune and Agaricus bisporus. Mycol Res 103: 635-640

Mulder GH, Wessels JGH (1986) Molecular cloning of RNAs differentially expressed in monokaryons and dikaryons of Schizophyllum commune in relation to fruiting. Exp Mycol 10: 214-227

Penas MM, Asgeirsdottir SA, Lasa I, Culianez-Macia FA, Pisabarro AG, Wessels JGH, Ramirez L (1998) Identification, characterization, and in situ detection of a fruit-body-specific hydrophobin of Pleurotus ostreatus. Appl Environ Microbiol 64: 4028-4034

Schuren FHJ, Wessels JGH (1990) Two genes specifically expressed in fruiting dikaryons of Schizophyllum commune: homologies with a gene not regulated by mating-type genes. Gene 90: 199-205

Van Wetter MA, Wosten HAB, Wessels JGH (2000a) SC3 and SC4 hydrophobins have distinct roles in formation of aerial structures in dikaryons of Schizophyllum commune. 
Mol Microbiol 36: 201-210

Van Wetter MA, Wosten HAB, Sietsma JH, Wessels JGH (2000b) Hydrophobin gene expression affects hyphal wall composition in Schizophyllum commune. Fungal Genet Biol 31: 99-104

Wessels JGH (1997) Hydrophobins: proteins that change the nature of the fungal surface. Adv Microbiol Physiol 38: 1-45

Wessels JGH (2000) Hydrophobins, unique fungal proteins. Mycologist 14: 153-159

Wosten HAB, Richter M, Willey JM (1999) Structural proteins involved in emergence of microbial aerial hyphae. Fungal Genet Biol 27: 153-160

Wosten HAB, De Vocht ML (2000) Hydrophobins, the fungal coat unraveled. Biochim Biophys Acta 1469: 79-86 
Figure legends

Fig. 1 Restriction map of the genomic DNA region containing the fv-hyd1gene. The entire nucleotide sequence of $f v$-hyd1was obtained by combining the determined sequences shown by arrows. Box represents the ORF with start and stop codons. Three closed boxes indicate introns. A solid line indicates a position of the 45c cDNA fragment obtained by differential display screening.

Fig. 2 Nucleotide sequence and deduced amino acid sequences of the fv-hyd1. Numbers show the nucleotide number starting from the start codon (+1). A coding region of 121 amino acid residues is shown by upper case letters, and three introns are shown by lower case letters. An open triangle shows a cleavage site of a putative signal sequence. Eight cysteine residues highly conserved among hydrophobins are circled. A TATA box-like sequence is boxed, and CT-rich regions are underlined. A polyadenylation signal was double-underlined. A closed triangle indicates a position where a polyA tail was added. The sequence has been deposited in the DDBJ database under accession number AB12668.

Fig. 3 Comparison of deduced amino acid sequences among fungal class I hydrophobins. Numbers show the amino acid residue number from the start codon. The amino acids shared five or more of the eight aligned sequences are black boxed. Asterisks indicate eight cysteine residues highly conserved among hydrophobins. The sequences used are from F. velutipes FVHYD1 (this work, AB12668), F. velutipes FVH1 (AB026721), P. ostreaus POH3 (Y16881), S. commune SC1 (X00788), Pholita nameko PDI263 (AB079129), C. cinereus COH1 (Y10627), L. edodes LE.HYD2 (AB079129), and A. bisporus ABH3 (Y14601). 
Fig. 4 Hydropathy plots of FV-HYD1 (A), FVH1 (B) and Pleurotus ostreatus POH3 (C). The plot was obtained by the parameters of Kyte and Dolite using a window of six amino acid residues. Asterisks indicate positions of conserved eight cysteine residues. The vertical arrows in each plot indicate the position of signal peptide cleavage site.

Fig. 5 Southern blot analysis of genomic DNAs. Genomic DNAs were prepared from $F$. velutipes dikaryotic strain MH092086 (lane 1 and 4), monokaryotic strain FB89 (lane 2 and 5), and monokaryotic strain MF38 (lane 3 and 6). Equal amounts of genomic DNA (10 $\mu \mathrm{g})$ were digested with Sal I (lane 1 to 3) or Eco RI (lane 4 to 6). The 45c cDNA fragment was used as a probe.

Fig. 6 Northern blot analysis of the fv-hyd1 transcript. Total RNAs were isolated from $F$. velutipes cells collected at different stages of fruiting body development; vegetative mycelia (lane 1) immediately before fruiting induction (mycelial mat scraping, water addition, and light exposure), at 4 hours (lane 2), on day 2 (lane 3), day 6 (lane 4), day 10 (lane 5, primordial stage), and day 16 (lane 6, mature-fruiting-body stage) after induction. Total RNAs were prepared from mycelial cultures on day 10 after subjected to only one stimulus out of the three treatments; light exposure (lane 7), water addition (lane 8), and mycelial mat scraping (lane 9). The 45c cDNA fragment was used as a probe for fv-hyd1. The 18S rDNA probe was also used to confirm an equal loading of RNAs on each lane. 


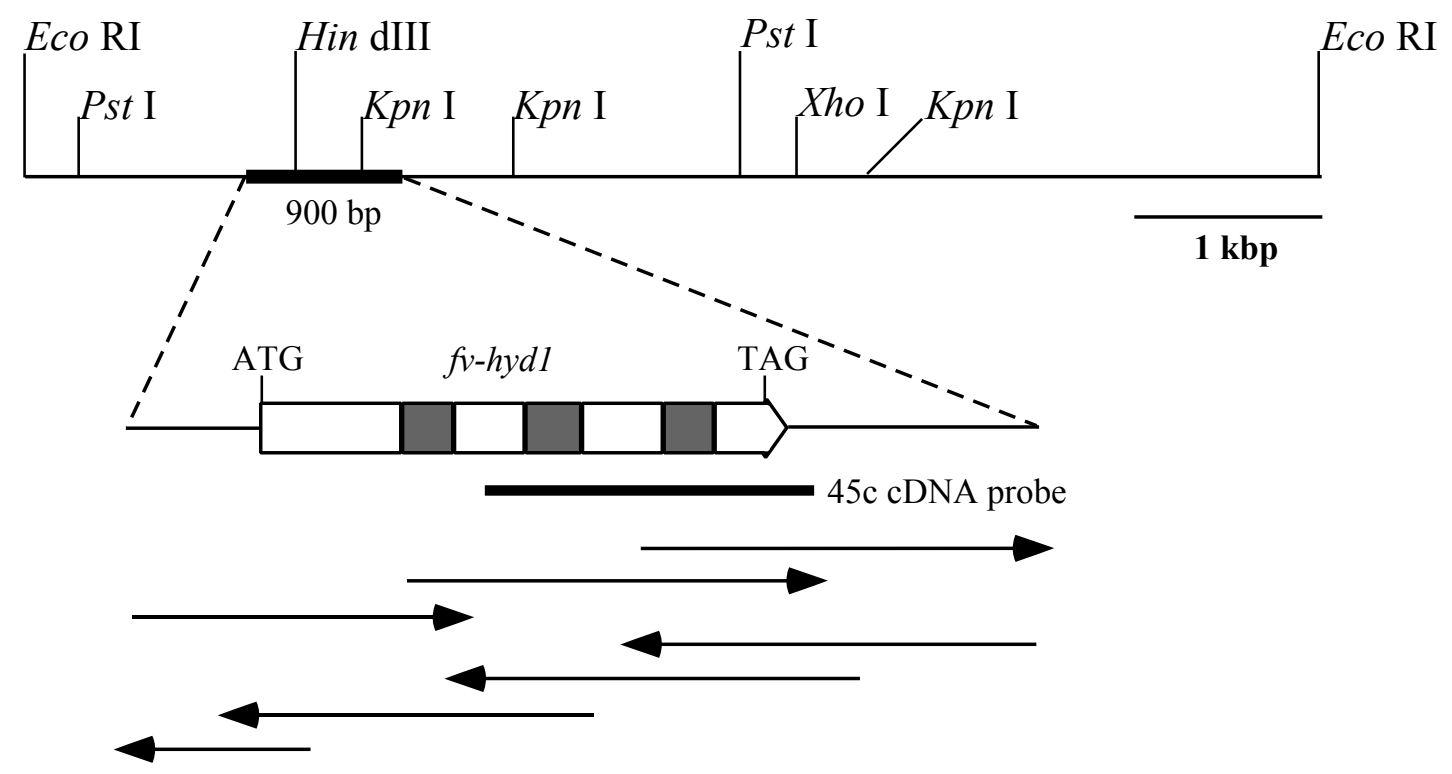

Fig. 1 Yamada et al. 
-240 TGGCCTGCGAAGTCCTCGGAAACGAGGAAACTTTATATTCGCGCCACCGTGACCAAGTGG

-180 TGGAACTATACTCATTTTCTTCCCCCTCATGCTTCCTGAACTGTAATTCCTGCTGTTCGC

-120 TCCACACGAGAATTTTGAGTATAAAGGCGCCTGGGTATTGCTGGTAAACTCATCAGCTCT

-60 TCCCTCCAGCAGATCTCCCACTCTCATCTCGACCAACTTCGTCTTTATAACTCGCTCATC

1 ATGAGACTCTCCATCTCCAAGCTTTCTGTAGTCATGTTGGCGCTGCCTATCCTTGCGTCC

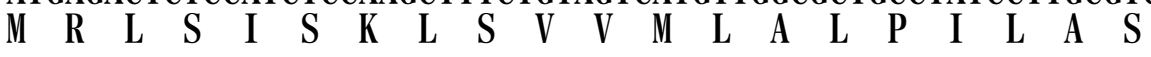

61 GCCAGCGTCTTGCCCCGCACTGGCAGTACCGGTGCTTCCTGTGGATCGACCACCTCTGGC $\mathrm{A}_{\triangle} \begin{array}{lllllllllllllllllll}\mathrm{S} & \mathrm{V} & \mathrm{L} & \mathrm{P} & \mathrm{R} & \mathrm{T} & \mathrm{G} & \mathrm{S} & \mathrm{T} & \mathrm{G} & \mathrm{A} & \mathrm{S} & \text { (C) } & \mathrm{G} & \mathrm{S} & \mathrm{T} & \mathrm{T} & \mathrm{S} & \mathbf{G}\end{array}$

121 CAAGCTCAATGCTGCAACAGCACCCAATCTAACgtgagcccatccegt cgtccetcccac

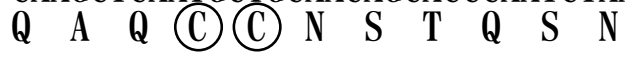

181 tagagatgggaat tgaccgaatgc tccagACGACCCCCTTGCCTTTCCCCCTCTCCGTAA $\begin{array}{llllllllllll}T & T & P & L & P & F & P & \text { L } & \text { S } & \text { V } & \text { I }\end{array}$

241 TCACTGGACTCTTGGGGATTGACCTCGGGGCTATCACTGGTCTTGTg taag t t catcg tc $\begin{array}{llllllllllllllllllllll}\text { T } & G & \text { L } & \text { L } & \text { G } & \text { I } & \text { D } & \text { L } & \text { G } & \text { A } & \text { I } & \text { T } & \text { G } & \text { L } & \text { V }\end{array}$

301 tcgggeccgtaatcatcagagt t taact tgaat t tctatccetccagTGGTTTGACTTGC G L T (C)

361 AACCCCATCACGGTCCTTGGCCTTGGAGGTACCAACTGCAACAGCCAGGCTGTCTGCTGC $\begin{array}{llllllllllllllllllll}N & P & I & T & V & L & G & L & G & G & T & N & \text { (C) } & N & S & Q & A & V & \text { (C) (C) }\end{array}$

421 ACCGGCAACACCTTCgtacgtcaatgggct tgtcgtgtcat tgat tgct tat tcct t T G N T F

481 ggctg tc tagAATGGTCTCATTAATTTGGGATGCTCCCCTCTCAACGTCGCCCTCTAGTG

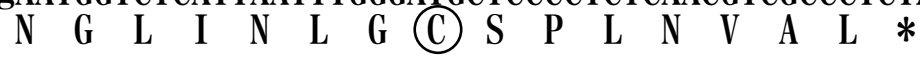

541 TACGGCGCCACCGATTGCTTTTAGCCTCGCGAGGTCGTCATTTTCTTCGTAACTTTCAAA

601 AATGCTCTGAAAACTTTATATACAAATGCATTTAGGACTGATATGTAGCTGTAGTATTCA

661 TACTTCACTGTTACATATCATTTTATTCTCT ACCTGTCGTGTGTGGCTTTACATATCTGG

721 TGCCCAATGTTGCTCTTCCCCGTGGTTTACTATCTTTCCTGTTAAGTGGAGTGACCCCTT 
FVHYD1

FVH1

$\mathrm{POH} 3$

$\mathrm{SC} 1$

PDI263

$\mathrm{COH} 1$

LEHYD2

$\mathrm{ABH} 3$

FVHYD1

FVH1

$\mathrm{POH} 3$

$\mathrm{SC} 1$

PDI263

$\mathrm{COH} 1$

LEHYD2

$\mathrm{ABH} 3$
* * * *

MRLS ISKLSVVMLALPILASASVLPRTGSTGA - - SCGSTTSGQAQCCNSTQSNTTPLPFPLS 60 -MVSFRAFTVAASLFATLAAATPLDTALPRAAD - - QC - - - NVSNQQCCNSVQQASSGPA - - - A 54 - - MFSRVIFCTFLILPLLAAATAIPRTNPPAP - - TC - - TTGSLQCCNSVQAASNPVV - - G 52 - - - - MRF SLAILALPVLAAATAVPRGGASK - - - - C- - - NSGPVQCCNTLVDTKDKHQ - - TN 48 - - MFARAASVFVLSLPILATANVLPRQD - - - - - NOC - - - NTGS LQCCSSVQSSSSSLV - - - A 49 - - - MQFKFLSTVALATLAVAAPAPTDPTPIPP - SQC - - NTGP IQCCNTVTQASNPVA - - G 52 - - - MQFKLAFVS IALATLAVATPAPRGEPA - - - SSC - - - STGDLQCCNTVEPASSPSA - - - S 50 -MFAR ISTIITTLFFAMLAAATAVPRTDP P PATGSOC-TAVGGDVNCCNSVQDASNDIV - - - G 58 VITGLLG IDLGA I TGLVGLTCNPITVLGLGGTNCNSQAVCCTGNTFNG-LINLGCSPLNVAL 121 L I L GLLGVVIQDVNVLVGLDCSP I TV I GGGNGGCNAS PVCCENNSFGS-LIS I GCVPIS I - - 113 LLAGLLG IVLGP I TGQVGLTCS P I TVI GVGGTSCSAQTVCCNGNSFNG-LIVVGCSPVNISI 113 IVGALLGLDLGSLTGLAGVNCSPVSVI GVGGNSCSTQTVCCEGTQFNG-LVNVGCTPINVGL 109 I LLGLLGVAÄGGLTGQVVGVTCSPITVIGVSGTSCSEQPVCCTGNTFNG-VIATGCTPVNVNL 110 LLLGLLG IVLQDLNVLVGLTCSP IS I I GL PGNSCNAQPVCCQNNNFNG-LIA I GCTP IN INL 113 T I LGLLG I VI QGVDVLVGLTCS P I TV I GLESGGCSAQAVCCTDNSNGG-L IS IGCLPVTL - - 109 LLAGLLG I VLGP I QGLVGLTCNP ISVIG-GGNSCSSQTVCCTGNNFS GGLLV I GCS INIDL 119

Fig. 3 Yamada et al. 


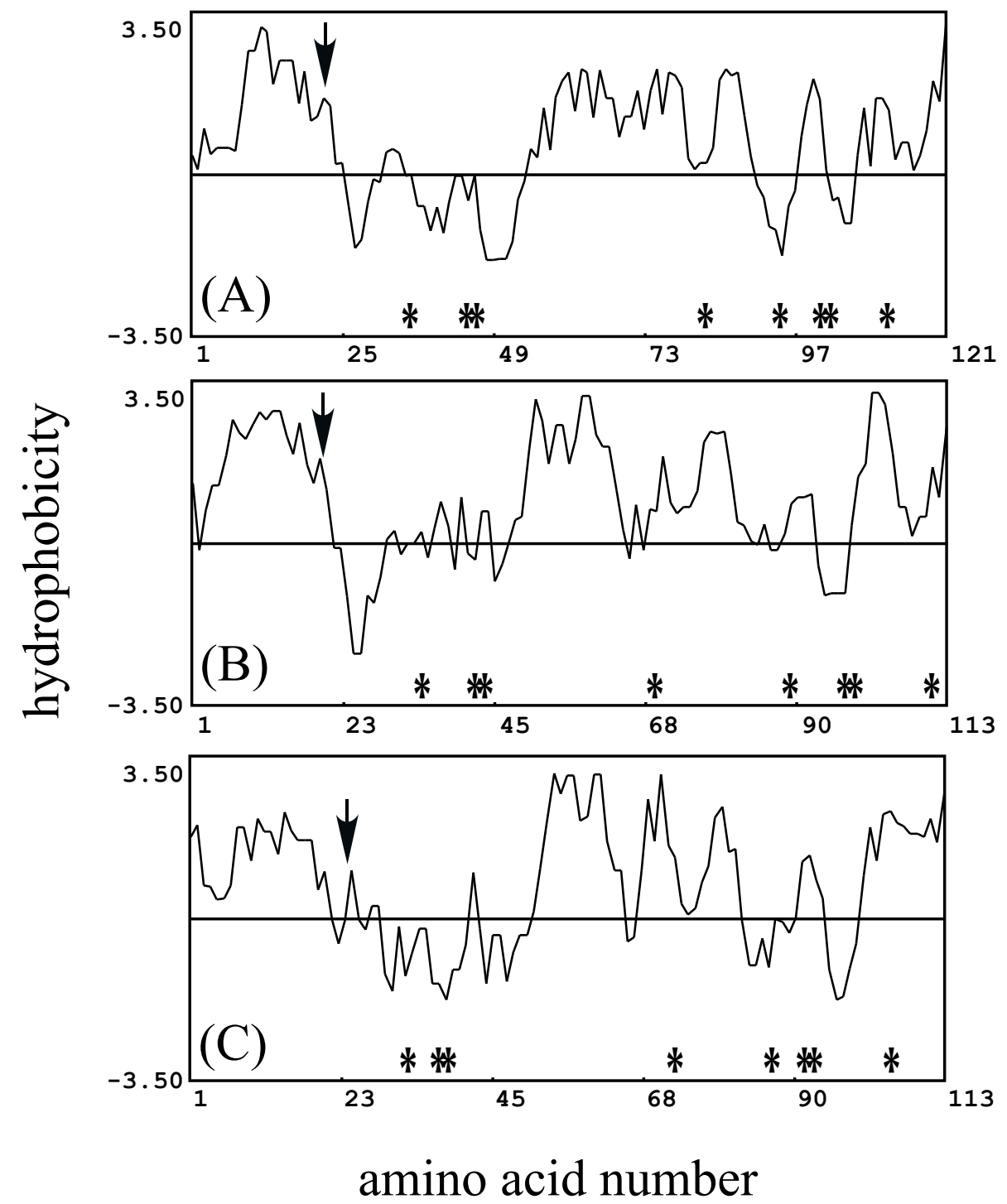

Fig. 4 Yamada et al. 


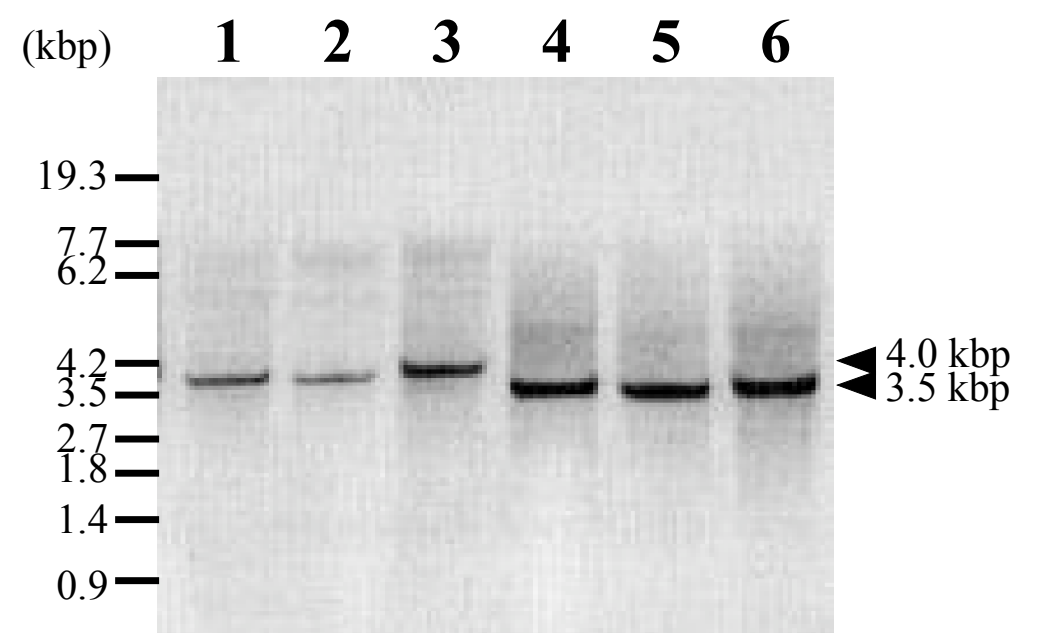

Fig. 5 Yamada et al. 

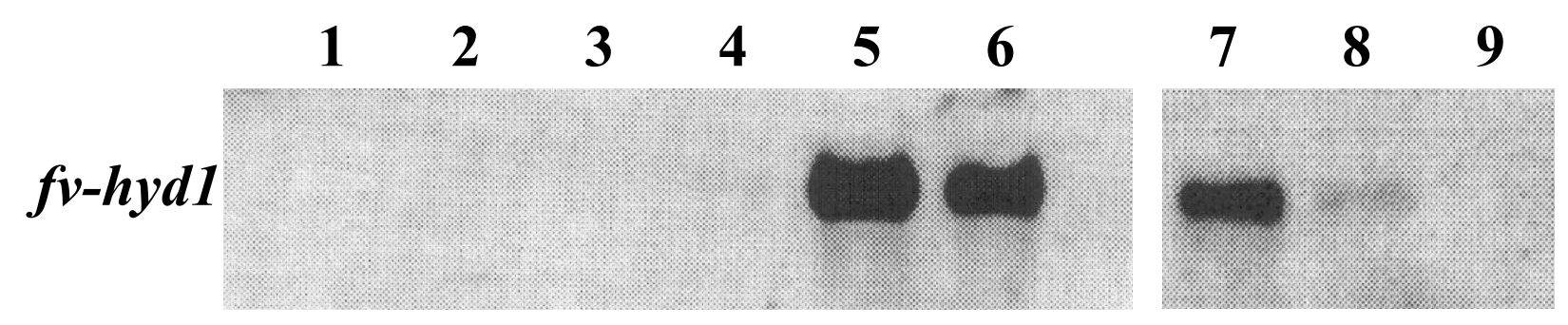

18S rRNA

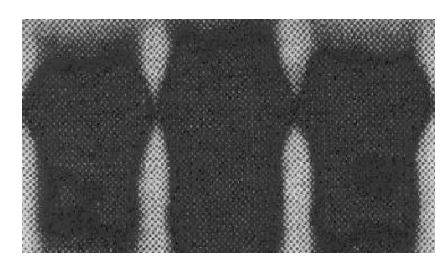

Fig. 6 Yamada et al. 